\title{
Faut-il protéger le métro des voyageurs? Ou l'appréhension du voyageur par les ingénieurs et les conducteurs
}

Robin Foot

Résumé : L'automatisation actuelle du métro parisien soulève un paradoxe moral étrange: dès lors que l'on supprime les conducteurs, les ingénieurs conçoivent un métro « protégé » des voyageurs mais ils laissent les métros avec conducteurs sans protection. Cette dissymétrie de traitement est d'autant plus étrange qu'elle semble trouver, du côté des conducteurs, un acquiescement tacite. La compréhension de cette situation étrange suppose d'analyser sur une relative longue période la manière dont les ingénieurs et les conducteurs composent leur communauté d'action au travers des processus d'automatisation, du travail et des conflits. Au cœur de cette dynamique, la peur de l'accident et du suicide voyageur apparaît comme un élément déterminant, même s'il reste la plupart du temps «inexprimé» sauf sous forme d'objet technique, dans l'élaboration d'une stratégie défensive du métier de la conduite. Summary, p.205. Resumen, p.205.

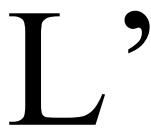

automatisation de la ligne 1 du métro parisien, prévue pour 2010, réactive la question ouverte par l'inauguration de Météor/ligne 14 en 1998 d'un traitement différencié de la relation aux voyageurs selon que le métro est exploité avec ou sans conducteurs. En effet, dès lors que l'on supprime les conducteurs, la mise en place de portes palières qui créent une séparation nette entre le quai et la voie semble s'imposer. Malgré les discours contraires qui affirment que ces portes palières

\footnotetext{
* Une première version, plus longue, de ce texte a été publiée dans les actes du 1er Colloque International de Psychodynamique et Psychopathologie du Travail qui s'est tenu à Paris les 30 et 31 janvier 1997.
} 
sont liées à la présence des voyageurs, à leurs suicides comme à leur chute ou descente sur voie, trois facteurs majeurs de perturbation du réseau, force est de constater que, en réalité, leur existence est liée aux conducteurs, à leur absence puisqu'il faut attendre ce nouveau projet d'automatisation de la conduite pour voir de nouvelles portes palières apparaître dans le métro. Ce projet, qui reprend pour l'essentiel l'architecture de Météor, nous donne l'occasion de faire retour sur cette histoire de l'automatisation de la conduite et, au travers elle, de suivre comment, au sein de la corporation ferroviaire de la RATP, des ingénieurs jusqu'aux conducteurs, l'appréhension du voyageur, au double sens physique et mental du terme, s'est réalisée dans le dernier quart du $\mathrm{XX}^{\circ}$ siècle.

Pour comprendre ce qui se joue dans cette confrontation entre deux techniques d'exploitation du métro, l'une avec conducteurs et sans porte palière et l'autre sans conducteur et avec porte palière, il nous faut suivre la manière dont les ingénieurs et conducteurs parlent de la conduite au travers aussi bien des processus d'automatisation que du travail ou des conflits. La symétrie que l'on tente d'établir entre conducteurs et ingénieurs ne signifie pas identité de traitement; elle doit nous permettre d'interroger le métro en circulant de la manière la plus continue possible dans les différents mondes des acteurs. Sans déroger à ce principe, on peut alors accorder plus d'im-portance dans l'analyse à l'un des acteurs, aux conducteurs par exemple, pour mieux explorer un point précis, la peur du voyageur dans ce cas. Suivre les acteurs oriente la recherche vers les "objets" qui "symétrisent" les points de vue. Ils deviennent les points de passage obligés de l'enquête. Nous analyserons donc les modes de mise en rapport et d'entredéfinition des acteurs et comment, au-delà des oppositions immédiatement perceptibles, se crée cette communauté ferroviaire.

\section{Automatisation du métro et principes de précaution des ingénieurs : une stratégie paradoxale.}

En 1979, avec la mise en place du pilotage automatique (PA) sur la ligne 5 , s'achève le processus de modernisation technique du réseau métro commencé en 1965. Dans le même temps, les responsables du métro s'interrogent sur les causes des incidents et accidents qui apparaissent dans l'exploitation. Ils constatent, de la part des conducteurs, des comportements qui mettent en cause la sécurité : franchissement de signaux fermés, non respect de procédure de marche en situation perturbée, non respect des règles de circulation en arrière gare... Si la plupart du 
temps les enquêtes mettent en évidence un comportement fautif $\mathrm{du}$ conducteur, les origines de cette nouvelle conduite, en rupture avec les représentations antérieures de la professionnalité reconnue des conducteurs, restent pour partie mystérieuse. Ce non respect de règles de sécurité, dans un milieu où elles constituent un indicateur de la professionnalité, est interprété comme le symptôme d'un trouble du groupe de conducteurs dans son rapport au travail. De leur côté, à la suite du constat d'un "sentiment d'ennui au travail", exprimé par les conducteurs et associé à la généralisation du pilotage automatique, le Syndicat Autonome Traction (SAT) demande à ce que sa catégorie retrouve, dans le travail, la qualification qu'elle a su inscrire malgré le PA dans les classifications par la grève d'octobre 1971. Cette revendication se focalise sur la restauration d'une habilitation pour les conducteurs à conduire en "manuel". Les explications des responsables convergent donc avec celles des syndicalistes: "Lorsqu'une ligne est équipée du pilotage automatique (...) au bout d'un certain temps (...) l'état d'esprit du conducteur se modifie. Il s'ennuie, s'interroge sur son utilité, parle de "baisse de sa qualification". Les services lui paraissent bien longs. Il peut penser de plus en plus à ses problèmes familiaux ou personnels, et sa vigilance a tendance à s'émousser. Il devient de plus en plus distrait (...)" ${ }^{1}$

\section{Un recul de l'automatisation : la conduite manuelle contrôlée}

La solution finalement retenue est le fruit d'expérimentations, entamées dès 1973 avec l'essai de couplage de la conduite manuelle contrôlée (CMC) avec le pilotage automatique sur la ligne 6, liées à un processus de concertation avec les représentants syndicaux. Elle se concrétise par un mouvement partiel de régression dans le processus d'automatisation. En effet, cette CMC constitue un mode intermédiaire entre celui qui existait antérieurement où aucun système de sécurité automatique n'était intégré [Foot, 1990: 136-137] et le PA où le conducteur est réduit, en dehors du service en station, à un rôle passif de surveillance d'un automate. Le conducteur assume donc de "nouvelles" responsabilités dans la conduite, en ce qui concerne en particulier les vitesses. Les

1 Rédacteur Alain Caire, Note sur le conducteur seul à bord de son train au métro de Paris, Note A2-401, présenté par le Métro de Paris, Direction du réseau ferré de la RATP, Service de l'exploitation, Comité permanent des métros sur pneumatique. $4^{\circ}$ symposium. Santiago. Avril 1979. 
dispositifs automatiques assurent alors seulement la surveillance du respect de la signalisation par les trains et $\mathrm{d}^{\prime}$ une présence consciente du conducteur avec le système de "l'homme mort". La CMC matérialise une conception traditionnelle de la professionnalité où la possibilité de faire une faute n'est pas interdite a priori par un dispositif technique, "en particulier les dépassements de vitesse sont toujours possibles". Le contrôle est renvoyé aux rapports hiérarchiques ; "il s'agit donc de sanctionner l'erreur plutôt que de la prévenir" 2 .

La CMC correspond à une reconnaissance par les ingénieurs des limites d'un automatisme non "autosuffisant" qui suppose de "maintenir quelqu'un derrière lui pour le surveiller en permanence" ${ }^{3}$. Pour les syndicats de conducteurs, elle permet de reconstituer la conduite comme base de la légitimité professionnelle. Ce mode de conduite, généralisé entre 1979 et 1985, signale également qu'une forme de redéfinition des domaines de compétences entre les conducteurs et les automates est envisageable sans que, a priori, les uns ou les autres pensent que cela nuise à la qualité du transport ${ }^{4}$.

C'est pour cela que dans les faits, sinon en théorie, le libre choix du mode de conduite, PA ou CMC, fut laissé aux conducteurs. Les conducteurs ne se sont pas emparés de la CMC pour se désennuyer ou pour se reprofessionnaliser comme certains le pensaient [Bouvier, 1985 : 127-128]. Elle n'est pas devenue le support d'une pratique collective unifiant le groupe qui aurait participé d'une recomposition identitaire mise à mal par la modernisation. Son introduction révèle au contraire le fractionnement du groupe dans ses pratiques: "Lorsqu'au mois d'août 1980, les conducteurs ont eu l'autorisation de conduire en CMC sur la ligne 13, nous nous attendions à ce que la majorité des conducteurs reprenne la conduite manuelle. Or ce n'est pas ce qui s'est passé : $42 \%$ seulement des services ont été assurés en CMC, totalement ou partiellement. Pourtant, j'avais - en tant que délégué - affiché dans le cadre syndical de Chatillon "que

2 Ibid.

3 André Pascal, adjoint au chef du service de l'exploitation à la direction du réseau ferré. Entre les lignes, nº76, Octobre 1984, RATP, p.17.

4 Cette absence de corrélation directe entre amélioration du transport et pilotage automatique (PA) était perceptible dès 1968 quand l'expérience du contrôle des temps de stationnement sur la ligne 7 permit une réduction sensible de l'intervalle entre rames et donc un accroissement des capacités d'offre sans que la ligne soit équipée en PA (cf. Foot, L'introduction de nouvelles techniques productives au sein des réseaux ferré et routier de la $\mathrm{RATP}$ de 1945 à 1985, Gip MI, 1987, pp.41-50). 
conduire en CMC c'était avant tout défendre sa qualification et prouver à la Direction ainsi qu'à certains techniciens avides d'automatisme intégral (type VAL) que l'homme peut, en fonction des progrès techniques, être opérationnel et rentable par ses valeurs professionnelles"" [Tract du SAT, mai 1981] .

Cinq ans plus tard, les pratiques des conducteurs d'une autre ligne expriment cette même tendance, certains l'utilisent, d'autres pas. De plus, parmi les premiers, les usages varient: un sondage effectué le 12 mai 1986 "révèle que sur 846 courses, $45 \%$ sont effectuées en $C M C^{\prime \prime}$ et que, alors que le PA est obligatoire quand l'intervalle entre deux rames est inférieur à deux minutes, c'est-à-dire en heures de pointe, "33,60\% des courses sont effectuées en CMC". Par contre, tandis que la CMC est obligatoire quand l'intervalle est supérieur à quatre minutes, près de $20 \%$ des courses sont alors réalisées en PA. Enfin quand le choix est libre, seulement $46,40 \%$ des courses sont effectuées en CMC, "ce qui est peu compte tenu du fait qu'un tiers des conducteurs enfreignent la réglementation en heure de pointe" [de Peslouan, 1987: 8-9]. Cette appropriation non seulement diversifiée mais surtout polarisée de la CMC, objet autorisant la conduite manuelle, considérée par certains sociologues comme la "justification du métier" [Lae, 1991 : 43], ne fit pas sens. Les pratiques de travail ne purent donc constituer un point d'appui à une mise en cause du PA. Celui-ci continuait à structurer l'imaginaire non seulement des dirigeants mais aussi des conducteurs. Les habitudes prises et les mentalités individuelles furent les principaux éléments convoqués pour expliquer le décalage constaté entre les discours des conducteurs et leur rapport effectif à la conduite. Un voile d'ignorance recouvrit dès lors ces usages qui mettaient à mal les justifications du compromis passé.

\section{Une reprise de l'automatisation et la question des portes palières}

Si cette résistance des pratiques de conduite à se conformer aux théories du métier et de la qualification ne semble pas susciter un grand intérêt, peut-être est-ce aussi dû à la réactivation du débat sur l'automatisation de la conduite au sein de la RATP, réactivation provoquée pour l'essentiel par les projets en province. En mai 1983, Lille inaugure avec succès son métro à petit gabarit et sans conducteur. Le VAL, dans un premier temps enfermé dans sa spécificité, ne semble pas pouvoir se constituer en référence pour le réseau parisien. L'automatisme intégral, 
est plus difficile à envisager sur un réseau ancien que sur un réseau neuf, sur un réseau "lourd " que sur un réseau « léger ». ${ }^{5}$ Mais le succès du VAL relance dans le milieu la problématique de l'automatisation. En 1985, ce sera le réseau lyonnais qui décide e transformer la ligne $\mathrm{D}$ en cours de construction en un métro sans conducteurs. La RATP participe humainement et financièrement à ce métro nommé Maggaly. Dès 1986, le projet d'automatisation intégrale du mouvement des trains (AIMT) devient un projet structurant pour les services d'études du réseau ferré et de la direction des équipements électriques, même si l'implication de la direction générale est limitée. A l'époque, il s'agit d'un projet global de modernisation concernant l'ensemble du réseau métro qui se traduirait donc, s'il était réalisé, par la suppression de la totalité des emplois de conducteurs et d'agents de terminus soit près de 4000 emplois [Villoutreix, 1990 : 215]. Avec les conflits de 1985 et surtout de 1986, cette perspective de suppression des conducteurs trouve un écho favorable auprès de nombre de responsables du réseau ferré. L'image de l'automatisation tend à s'enraciner dans celle de la qualité de service ; étayée par un argumentaire d'apparence technique, elle fait corps dès lors avec elle. Progressivement, au terme de ce processus, le métro acquiert enfin, dans l'imaginaire, la légèreté d'une "pure" technique désengagée de la pesanteur du social, de ses syndicats, ses négociations et ses conflits. Sans travailleurs, la production peut alors faire rêver véritablement les ingénieurs. Après l'AIMT, Météor participe de ce même fantasme, d'ailleurs récurrent dans le monde industriel: "Métro du $21^{e}$ siècle, Météor sera entièrement automatique. C'est-à-dire plus sûr, rapide, régulier, plus souple à exploiter, donc plus efficace, toutes conditions nécessaires à l'offre de qualité qu'attendent les voyageurs d'un transport moderne." 6

La grève du 20 décembre 1985, réitérée l'année suivante, s'est aussi diffractée dans l'imaginaire. Ces grèves de conducteurs participent de la création d'une relation d'évidence entre la présence d'humains et l'absence de transport. Mais pour que cet imaginaire social perdure, encore faut-il que sur le terrain l'automatisation devienne de plus en plus réelle. La participation des ingénieurs de la RATP au projet Maggaly procède de ce mouvement et

5 Pierre Beuchard, adjoint au chef du service des études de l'exploitation à la direction du réseau ferré. Entre les lignes, n76, Octobre 1984, RATP, p.13.

6 Plaquette "Opération Météor, le futur a son métro", (réalisé par le cabinet Roux Séguela Caysac \& Goudard) RATP. 
contribue à définir les options technologiques à retenir pour une ligne "sans conducteurs". En particulier, ils ne reprennent pas le choix fait pour la ligne D par les responsables lyonnais qui ont, pour des raisons de coûts, opté pour une solution qui n'assure pas une "étanchéité" parfaite entre le quai et la voie. A Paris, les responsables considèrent qu'ils ne peuvent prendre ce risque: " $A u$ premier accident voyageur où un métro automatique serait impliqué, vous imaginez le résultat le lendemain dans la presse. Immédiatement on aurait les politiques sur le dos qui nous dirait de prendre des mesures pour que cela ne se reproduise pas" (cadre dirigeant du réseau ferré, 1988) ${ }^{7}$. Comme à Lille, des portes palières, installées en bordure des quais, semblent être la seule solution qui assure "une protection totale" ${ }^{8}$ : " "Chaque année, sur le réseau parisien, il se produit 120 à 130 suicides contre 50 il y a dix ans " admet Pierre Lecompte, des services techniques de la RATP. "C'est pourquoi nous expérimenterons au début de 1989 des portes palières à la station Place des fêtes ${ }^{\prime \prime}$.

Protéger les voyageurs sur le quai et protéger la voie contre les intrusions intempestives tel est le "script" [Akrich, 1987] qui, avant d'être objectivé par des portes palières, est défini par le cahier des charges de Météor: "la protection des voyageurs sur un quai est essentiellement réalisée par un isolement physique total du quai par rapport à la voie, permettant d'empêcher les chutes et les intrusions de personnes en voie, principales causes actuelles de perturbation du trafic sur le réseau parisien." ${ }^{10}$ Les fonctions auxquelles doivent répondre ces portes palières se définissent de plus en plus précisément: "Le but des façades de quai est d'isoler totalement le quai de la voie. Pour cela, la fermeture du quai doit être réalisée sur toute la longueur du quai et sur une hauteur suffisante pour éviter que la façade puisse être "enjambée" (...) Les façades doivent résister à une poussée de la foule sans déformation ni rupture." 11 Mais il ne s'agit pas seulement de contenir les voyageurs, encore faut-il leur permettre de prendre le métro, de "ménager des sas" entre ces "deux univers clos" que constituent la rame et les quais équipés de portes palières. Ces sas "matérialisés par les portes du train, les portes palières et l'espace inter-portes"

7 Extrait d'un entretien avec un responsable lors d'une restitution d'une enquête faite sur le projet Maggaly de Lyon à la demande de la RATP.

8 “AIMT, le temps de la réflexion", Entre les lignes, n¹13, RATP, p.15.

9 Antoine Bonduelle, "Des métros sans conducteur!" Science et Technologie, n¹0, novembre 1988 .

10 Matra Transport, 20/7/90, AIMT. Spécifications des besoins fonctionnels, p.157.

11 Matra, op. cit. P.160. 
doivent "empêcher l'entrainement des voyageurs par le train (...) En particulier, il est primordial de garantir l'immobilité totale du train pendant le transfert, de façon à empêcher l'effet "guillotine" qui résulterait du coincement d'un passager entre les différentes portes au moment de la mise en mouvement du train."

Ce programme "de protection des voyageurs sur les quais" et de prévention "de l'entraînement ou l'étranglement d'un voyageur, d'une main coincée ou serrée" est l'expression d'une morale professionnelle fondée sur le souci de la conservation de l'intégrité des personnes qui s'incarne, "grâce à l'ingénieur, dans la nature des choses" [Latour, 1993 : 30]. Dans ce processus d'automatisation, l'accomplissement de la morale professionnelle est déléguée aux portes palières. À charge pour elles, désormais, de réaliser ce programme moral.

Outre le coût financier [Villoutreix, 1990 : 211], des problèmes techniques subsistent. En particulier dans le traitement des stations en courbe. Le fait que quelqu'un puisse rester entre la rame et les portes palières, au démarrage du métro, est une source d'incertitude majeure. Tout traitement technique de cet espace induit non seulement un surcoût - certaines analyses parlaient du doublement de l'investissement - mais surtout un risque de perte de fiabilité et de disponibilité du système dans son ensemble. Le processus par lequel la morale professionnelle de l'ingénieur peut se réaliser est complexe et interactif. $\mathrm{D}^{\prime}$ autant qu'il ne se déploie pas dans le seul monde des objets. D'autres interactions sociales viennent perturber cette recherche.

En effet, l'expérience de passage d'une ligne existante, la ligne 11 en l'occurrence, en automatisme "intégral" est abandonnée. Le projet d'AIMT est intégré dans Météor. Cette nouvelle orientation manifeste la réunification des stratégies particulières - celles du réseau ferré et des services techniques - sous l'autorité de la direction générale. Cette remise en ordre de la RATP est un des axes majeurs de la réforme à laquelle a procédé Christian Blanc à son arrivée en 1989. Elle formalise également que l'automatisation du réseau existant, c'est-àdire la suppression des conducteurs n'est plus, dans l'immédiat, à l'ordre du jour.

Même si la question des accidents voyageurs dans le processus d'automatisation intégrale du mouvement des trains n'a pas été à lui seul déterminant, il a contribué à 
infléchir la trajectoire du réseau. Le projet d'AIMT est venu buter sur les "portes palières". Expression matérielle des "principes de précaution" et de la culture de prudence en vigueur dans le monde ferroviaire où l'objet technique "doit être sécuritaire au sens ferroviaire du terme; c'est-à-dire qu'il ne doit engendrer aucune action pouvant compromettre la sécurité des personnes transportées" 12 , ces portes palières constituent l'élément de clôture nécessaire au système de gestion automatique des trains. Elles y ajoutent leur part de complexification et de surcoût. Cela suffit, pour qu'au moment où une nouvelle stratégie de la RATP se dessine, elles aident à faire échouer le projet initial.

Il est aussi conforme à cette idéologie de la "sécurité intrinsèque" du système d'objets de reporter sur les agents les zones d'incertitude en matière de sécurité. C'est le fameux principe ferroviaire du "lampiste", de ce cheminot que l'on envoie avec sa lampe à la main pour assurer la sécurité des circulations quand la signalisation fait défaut. Sur lui repose dès lors toute la sécurité. Avec l'abandon de l'AIMT et l'adoption du projet Météor, la question de la prévention de la chute des voyageurs sur la voie reste donc en l'état. Il revient toujours aux conducteurs d'assumer cette fonction.

\section{La réglementation et la conduite centrale pour isoler les conducteurs de la hiérarchie et des voyageurs}

En 1984, sur une ligne équipée de matériel pneu, la CMC vient d'être installée mais, pour l'instant, seule une voie est équipée de rétroviseurs permettant au conducteur de faire, quand il est en conduite manuelle, le service en station assis sur son siège, au centre de la loge. Cette modification, peu spectaculaire, car elle n'engage que des objets "ordinaires", un rétroviseur et un siège, introduit pourtant une transformation importante de la mobilisation du corps dans le travail du conducteur, et la manière dont il s'engage physiquement pour passer de la conduite au service en station.

En effet, avec le PA, si le conducteur peut être assis à l'entrée en station et au début du "service des portes" en station, il doit, pour la fermeture des portes et la sortie de la station, adopter la gestuelle de l'ancien chef de train. Après

12 Claude Hennebert, adjoint au chef du service des études à la direction des services techniques. Entre les lignes, nº76, Octobre 1984, RATP, p.15. 
s'être assuré que la signalisation l'y autorise, le conducteur se retourne, sort légèrement le buste de la loge, surveille la fermeture des portes et enclenche le PA. Dans cette position, il peut vérifier que, lors du dégagement de la rame, aucun voyageur ne reste accroché. Dans ce cas, le rapport au voyageur se fait sans la médiation d'objets techniques par l'engagement du corps dans la vision de la situation, de l'état des quais. Le mode de conduite centrale bouleverse cette relation: " portes de cabines fermées et non manoeuvrables de l'extérieur par les voyageurs, (il) implique un réajustement des points d'arrêt PA et l'installation de rétroviseurs ou de téléviseurs au tympan des stations. Le siège du conducteur est remis au centre de la cabine et la commande "fermeture-départ" est installée au pupitre. Le conducteur peut alors, s'il le désire, rester assis à son poste pendant le départ, la marche et l'arrêt du train" ${ }^{13}$.

Par la fermeture des portes et la technicisation du regard porté sur les voyageurs, la conduite centrale participe d'une recomposition du rapport au voyageur. La surveillance passe dorénavant par la médiation d'un objet, rétroviseur ou moniteur, implanté sur le quai, au niveau de la tête du train. En conduite centrale, donc, dès le départ, le conducteur perd de vue ses outils de vision du train. Lui part tandis que sur le quai restent les images d'un train qui s'en va. Dépourvu d'images, il reste au conducteur de conduire en aveugle son dégagement du quai. L'agencement de conduite, plutôt que d'équiper la vision du conducteur dans son mouvement, instrumente, par la réglementation, de fatalisme ses sens: "Assurer le maintien de la fermeture des portes jusqu'à dégagement complet du quai par le train et exercer en même temps, dans la mesure du possible, une surveillance visuelle et auditive très attentive." (Article 09-13 de l'instruction F36. Souligné par nous). Ce dans la mesure $d u$ possible exprime l'incertitude quant aux conditions de sécurité d'un départ dont personne, ni les producteurs de ce règlement ni les conducteurs qui sont censés l'appliquer, ne sait exactement comment mesurer ce possible. Mais dans cette écriture réglementaire se lit également que suivant sa place, l'effet est loin d'être symétrique. Pour le conducteur, le flou de l'expression combinée au dispositif de conduite - conduite centrale et rétroviseur - ne l'aide pas à accomplir en sécurité son travail. L'incertitude "vue" de la place du conducteur, loin de lui offrir un espace pour renforcer sa position dans les

13 Rapporteur G. Teilhout, Note sur le conducteur seul à bord d'un train en pilotage automatique, op. cit., p.11. 
rapports hiérarchiques, correspond à un espace contraint où le règlement instrumente l'imprévisibilité des situations afin que le conducteur soit amené à en assumer la responsabilité.

Pour les auteurs du règlement, cet article est censé éviter qu'un accident voyageur, en station, puisse être imputé comme faute, dans un procès, à d'autres qu'au conducteur. En effet, l'action de l'encadrement hiérarchique ne peut se démultiplier que s'il parvient à "localiser activement les interactions" des agents qu'il commande en créant "un ensemble de partitions, de cadres, de paravents, de coupe-feu" [Latour, 1994: 594]. Par la production d'une réglementation, d'une prescription du travail et des objets techniques, l'encadrement détermine non seulement le domaine de compétence de l'agent mais aussi le sien. Pour poursuivre ce que dit Bruno Latour, la force de l'énoncé "dans la mesure $d u$ possible" tient à son efficacité comme coupe-feu qui permet d'isoler les conducteurs de la hiérarchie.

\section{La conduite manuelle contrôlée et les pratiques de conduite}

L'analyse de l'appropriation de la CMC permet de percevoir ce qui trame, pour le conducteur, le rapport à la conduite et au service voyageur. Se rapprocher de la conduite manuelle ou prendre position en conduite centrale, c'est se distancier des voyageurs, du service des portes. Dans les pratiques de conduite, les deux ont parties liées. Dans les postures et attitudes de chacun, se lit l'articulation réalisée par le travail du corps entre le mouvement des trains et le mouvement des voyageurs. Certains vont adopter des attitudes clairement "déviantes" par rapport aux règles jusque là en usage dans le métier, tourner le dos à la voie ou lire, comme si, sans le dire, ils rendaient visibles aux autres conducteurs mais aussi à la maîtrise qu'ils ne veulent plus voir l'entrée en station, qu'ils ne supportent plus ces arrivées. Comportements déviants connus au sein du groupe des conducteurs d'une ligne, mais tus. A l'opposé, d'autres vont tenter, par l'usage de la $\mathrm{CMC}$, de restaurer un relation active au danger, de ne plus se regarder "être transportés" [de Peslouan, 1987 : 32]. Alors, contrairement aux préconisations, ceux-là vont utiliser la $\mathrm{CMC}$ en heure de pointe, au moment où l'affluence augmente le risque [de Peslouan, 1987 : 29 ; Foot, 1990 : 146147]. 
Outre la vigilance accrue que ce mode de conduite induit, parce qu'il affranchit le conducteur du contrôle des vitesses, il lui permet également de réguler ses appréhensions en entrée de station en régulant son allure quitte pour cela à rouler en dessous de la vitesse prescrite et donc à déroger aux règles ferroviaires ${ }^{14}$. La capacité de freiner à temps dépend en effet pour une large part de la vitesse de réaction du conducteur certes mais aussi, et surtout, de la vitesse du train. En effet, l'énergie que l'on doit dissiper si l'on veut stopper un train est liée au carré de sa vitesse $\left(E=1 / 2 \mathrm{mv}^{2}\right)$. Toute variation dans la vitesse $d u$ train a donc des répercussions extrêmement fortes sur l'efficacité technique des pratiques de conduite. De cette efficacité dépend la construction dans la durée de "savoirfaire de prudence" [Cru, 1985: 50] où s'agencent, en dynamique, autour de la peur, la conduite de l'agent et celle $\mathrm{du}$ train. Exprimer sa peur peut alors être une ressource pour se réapproprier le travail [Cru, $1997: 8$ ]. L'alternative entre conduite en CMC ou PA met chaque conducteur dans cet espace où il est amené à choisir entre accepter d'être agent de sa peur ou d'accepter d'en être le patient ${ }^{15}$.

Dans le processus de recomposition de la technique de conduite, le groupe conducteur n'a pas, semble-t-il, au début des années 80 , reconstruit de "stratégie collective de défense" [Dejours, 1993: 57]. Dans les entretiens avec les sociologues, si ce n'est dans l'espace du travail, la peur du suicide et de l'accident s'exprime. Sur 35 entretiens de conducteurs au cours d'une enquête effectuée en 1984, tous évoquent le suicide pour caractériser la spécificité de leur travail. A l'époque, nul besoin de questions directes pour que la diversité des rapports aux suicides apparaissent dans les discours. Hormis les agents de station, tous les autres

14 Dans la réglementation ferroviaire, les indications de vitesse sont des prescriptions d'allure et non pas des vitesses limites à ne pas dépasser. Un conducteur est, en théorie, si rien ne s'y oppose tenu de rouler aux vitesses prescrites.

15 Les couples action/passion et agent/patient nous semblent pertinents pour pouvoir rendre compte non seulement des différents points de vue dans le cours d'une action mais aussi de la posture d'une personne mise en situation d'agir. Que dans une situation donnée, un agent ne parvienne pas à transformer la violence d'une action qui s'exerce sur lui autrement qu'en prenant sur soi et il se transforme en patient c'est-à-dire en homme souffrant d'une passion qui le tient à sa place. Un OS sur chaîne en constitue un archétype. Qu'au contraire, il parvienne à faire passer l'action de l'autre sur lui dans l'objet de son travail, que par son travail il puisse canaliser la force de la mise en situation et alors la violence se sublime c'està-dire qu'elle "ménage des ouvertures nouvelles à la dialectique désir-souffrance" [Dejours, 1988 : 21] et, de sa place, celui qui était menacé d'être transformé en patient recouvre son statut d'agent. 
agents, inscrits dans la régulation ferroviaire que ce soit au terminus, en ligne ou au poste de commande central, l'évoquent peu ou prou, même si les manières d'en parler diffèrent. En particulier entre conducteurs et régulateurs du poste de commande central. Pour les premiers: "le suicide désigne un ensemble de rapports au public, à l'entrée en station, à une bousculade, un accident, un acte volontaire. Le suicide est, pour le conducteur, l'aboutissement ultime, quand il se concrétise, d'une somme d'appréhensions, de frayeurs maintes fois répétées à chaque course". Pour les seconds: "le suicide est devenu un terme générique de l'ensemble des incidents et accidents provoquant une interruption importante de la circulation des métros, cela se traduit par la phrase, au moment de la relève, échangée entre les chefs de régulation: "alors, aujourd'hui, pas de suicide " [Foot, Rajoharison, 1985]. Cette importance du suicide dans l'espace de travail ne date pas des années 80 mais se manifeste déjà dans l'univers du métro à la fin des années 70 [Bouvier, 1978 : 32-33].

\section{La mort d'un voyageur et les solidarités du groupe conducteur}

Au début des années 80 , cette peur du suicide, sauf en marge du travail, n'est pas reprise dans la "langue de métier". Dans cette ambiance, un fait crée rupture.En novembre 1984, au départ d'une gare, en heure creuse, un train entraîne un voyageur qui avait tenté de monter après le signal sonore. Celui-ci meurt. Une plainte est déposée par sa famille. Le jeudi 19 décembre 1985, le conducteur de la ligne A du RER est condamné à 6 mois de prison avec sursis et $3000 \mathrm{~F}$ d'amende par le tribunal d'instance de Meaux. Le vendredi 20, la grève appelée par les syndicats sur les lignes $\mathrm{A}$ et $\mathrm{B}$ du RER s'étend rapidement à l'ensemble du réseau. Les conducteurs du métro, sans s'occuper de la loi du 31 juillet 1963 qui impose le dépôt d'un préavis de cinq jours francs avant la grève, font corps avec le conducteur condamné. Pour la première fois, une grève de cette ampleur se produit de manière sauvage et, qui plus est, le jour du départ pour les vacances de Noël. Ces deux faits, en rupture avec les modes de régulation traditionnels de la conflictualité dans le réseau ferré, signalent que quelque chose d'extraordinaire se produit. A suivre le déroulement de cette grève, on perçoit les points par lesquels chaque conducteur s'est identifié au conducteur "condamné", effaçant ainsi les distinctions de réseaux. Ceux du RER et ceux de "l'urbain" se sont retrouvés dans ce procès et cette condamnation. 
A l'examen, le conflit vise non pas la décision de justice mais l'attitude de la direction et de la hiérarchie par rapport à "la faute" d'un conducteur. En effet, un des éléments de résolution du conflit se joue autour de la restauration d'une solidarité entre la direction et le conducteur : la direction s'engage dorénavant à soutenir le conducteur en appel et accepte la décision du tribunal des prud'hommes, instance juridique où salariés et employeurs sont à parité de grandeur, pour la sanction administrative. Avant le conflit, l'attitude de la direction et de l'encadrement exprimait que ce procès n'était pas leur histoire, que la faute du conducteur - non respect de la procédure réglementaire liant la fermeture des portes au déclenchement du bruiteur - n'engageait ni l'institution ni eux-mêmes. Le seul acte de l'institution a été la mise à disposition d'un avocat pour l'accusé: "Au moment $d u$ procès, il y avait une délégation de plusieurs centaines d'agents de la RATP. Il y avait l'avocat de la RATP. Et pas un cadre, personne de l'encadrement si petit soit-il. Uniquement du personnel d'exécution. Dans la salle du tribunal, ce qui est compréhensible, le personnel s'est dit: "on se fout de nous. L'entreprise, la direction, tout le monde se fout de nous. " (...) L'avocat de la RATP qui intervenait en général dans ces affaires a bien plaidé dans le sens de trouver des excuses, de minimiser l'affaire mais personne de la direction (...) Il y a eu un sentiment "on se moque de nous, on nous méprise", un sentiment d'abandon" (Claude Quin, Président de la RATP, 1981-1986, entretien en 1997).

La réaction des conducteurs exprime qu'ils jugent que le travail ne peut être isolé des rapports hiérarchiques et de l'organisation. L'existence d'une justice prud'homale rappelle que le contrat de travail "est le seul contrat civil défini juridiquement par l'inégalité des cocontractants. [Grumbach, 1978: 5]. Cette inégalité économique des parties se traduit, pour le salarié, par un abandon temporaire de sa "souveraineté" qu'il confie, pour un temps, à l'employeur : "La soumission du travailleur à l'autorité de l'employeur constitue ainsi la caractéristique "essentielle" du contrat de travail" [Supiot, 1994: 113]. L'espace du travail est par conséquent un espace de subordination dans lequel les délégations de responsabilité sont formalisées. Le jugement d'un salarié doit donc interroger "la partie technique de son travail, son organisation et les structures du commandement (...) Le salarié ne doit plus être "objectivé" par la faute commise, mais au contraire "subjectivé" par la mise à jour de l'environnement et de l'histoire du contrat" [Grumbach, 1978: 36 et 42]. Par cette grève, les conducteurs ne nient pas la mort d'un homme 
mais refusent la désolidarisation de l'encadrement. C'est à partir de la compréhension de la façon dont le dispositif productif oriente le travail et lui donne une "propension" à se réaliser sous certaines formes que l'on peut qualifier un événement comme faute d'un agent ou comme forme limite des pratiques ordinaires c'est-à-dire inscrite dans les limites de ce qui concoure à cadrer l'action.

\section{Le conflit entre savoir faire de prudence et qualité de service}

Dans le conflit autour de la qualification d'une pratique comme "faute" ou comme "ordinaire", se joue le mode de partition du domaine de compétence dans la conduite entre conducteurs et l'encadrement. Dans les faits, par le procès et le conflit, direction et syndicats redéfinissent les manières de limiter et de cadrer par des objets - depuis le dispositif de conduite centrale jusqu'aux lignes de peinture en passant par la production de textes et des paroles, l'espace dans lequel les pratiques de travail sont légitimes et où, par conséquent, la direction assume la responsabilité des pratiques de travail. La mise en cause de la réglementation et des stratégies de régulation basées sur le contrôle des temps de stationnement sont les marques que définir l'acteur à qui imputer une action ne va pas de soi [Callon, 1991 : 206]. Cela suppose de tester la résistance des liens qui solidarisent les différents éléments de l'agencement productif. Dans cette épreuve, la note d'août 1985 — édictée donc après l'accident de novembre 1984 -, qui indiquait que les conducteurs ne devaient "pas tenir compte des mouvements résiduels de voyageurs" afin d'améliorer l'offre de places par réduction des temps de stationnement, manifeste, comme l'absence de cadres au procès, la rupture entre les conducteurs et les cadres. Cette note est le premier élément de l'agencement à céder dans le conflit. Elle est suspendue dès le 23 décembre 1985. Si, par contre, la direction du réseau ferré maintient la réglementation concernant le service des portes, elle ouvre des négociations avec les syndicats pour étudier à quelles conditions celle-ci peut être respectée.

En particulier, se pose la question de savoir comment peuvent être conciliés les objectifs d'augmentation de l'offre sur la ligne $A$ du RER, pour résoudre le problème de sa saturation, avec le respect de l'article 09-09 qui stipule que "le signal de départ ne doit être donné que si la descente et la montée des voyageurs sont terminées et toutes les conditions préalables prescrites, notamment celles de sécurité, sont 
remplies". En effet, respecter cet article revient dans les faits à revenir à une conception d'une production dépendante de la régulation des voyageurs, du contrôle de leur flux. Celle justement qui s'est trouvée mise en défaut au moment de la modernisation du métro et auquel s'est substitué, au début des années 70, le contrôle du temps de stationnement des rames. Même si le rapport entre stationnement et mouvement des trains se pose dans des termes différents sur le RER et sur l'urbain, compte tenu de la saturation de la ligne A, l'acuité de la question est extrême. Afin de tenter de résoudre la saturation de la ligne $\mathrm{A}$, il faut aller plus vite et pour cela mieux gérer les arrêts, en l'absence de modification du système technique. Pour éviter qu'une dérégulation de la ligne A ne provoque un écroulement de l'ordonnancement des rames avec ses conséquences en termes de dégradation de la qualité du transport et ses répercussions sur l'activité économique de la Région, le dispositif productif focalise alors l'attention des conducteurs sur la régulation de l'allure en interstation et le contrôle des temps de stationnement. La saturation de la ligne A suppose une gestion tendue des flux. Cette tension s'exerce au premier chef sur les conducteurs [Clot \& alii, 1990].

Au quotidien, les conducteurs sont soumis, au nom de la qualité de service et du respect du voyageur, à minimiser le ralentissement occasionné au "carrousel" des trains par le stationnement. Il s'agit donc de rentrer en station aux vitesses permises par le profil de la voie, de limiter le temps de stationnement à celui qui permet à l'essentiel des échanges quai/voie de se faire et, enfin, de dégager au plus vite. Dans cette contrainte de gestion, les "savoir-faire de prudence" ne peuvent guère trouver à se former. A peine permis par l'introduction de la CMC sur l'urbain, ils sont contrariés par les impératifs productifs définis par la hiérarchie. Sur le RER où, à l'époque n'existe pas de système de pilotage automatique, le conducteur est sommé de résoudre par les gestes auxquels il consent volontairement ce conflit entre le sens du travail pour lui, fait aussi de la peur des accidents, et le sens du travail pour autrui tel qu'il est exprimé par la hiérarchie et les voyageurs. Cette saisie en "double bind" du conducteur est toujours agissante, en particulier aux heures de pointe : "on ne peut pas respecter les règles sur la fermeture des portes ... on ne peut pas respecter le règlement à la lettre, si on devait respecter 
vraiment les règles de sécurité entre 18:00 et 19:00, on ne partirait jamais" (conducteur 1993. MC. p.88) ${ }^{16}$.

Ainsi réinscrite dans des pratiques ordinaires, la faute du conducteur de la ligne A prend un autre sens. Elle matérialise le fait que le rapport à la conduite et au service voyageur se structure essentiellement dans les heures de pointe, là où les tensions sont extrêmes. Quand cette structure ne semble plus nécessaire en heure creuse, ses effets perdurent. Les distinctions entre les heures de pointe et les heures creuses ne peuvent certainement pas s'accomplir sur le mode d'une rupture entre une conduite fataliste et une conduite prudente. L'usage diversifié de la CMC l'avait déjà montré : "certains conducteurs vont même jusqu'à alterner les deux modes de conduite sur la même course: en CMC sur les tronçons de ligne chargés, en PA pour les dernières station $d u$ sud qui sont très peu chargées, afin de "récupérer" [de Peslouan, 1987 : 29]. L'heure creuse est remplie de ce qui a nourri l'heure de pointe. Le refus de la qualification d'une pratique de travail comme faute relève de ce que le rapport des agents au temps de travail ne se fait pas sur un mode discret mais continu. C'est pour cette raison aussi que le procès de l'un a été ressenti comme la mise en accusation de tous.

De part et d'autre des pratiques, par les luttes, la réglementation, les innovations techniques et les procès se joue la définition de l'agencement à qui, du conducteur ou de la hiérarchie, peut être imputée une faute. Pour reprendre la formulation de Jacques Girin [Girin, 1995] : qui est habilité à répondre de l'agencement? Quelle est la nature de son mandat? A la naissance du métro, une réponse partielle avait déjà été donnée: "Les travailleurs occupés de l'entreprise seront assurés contre les accidents aux frais exclusifs du concessionnaire, qui ne pourra faire de ce chef, aucune retenue sur les salaires. De plus quel que soit l'imputation de la responsabilité d'un accident, le concessionnaire sera toujours directement responsable vis-à-vis de la victime du paiement de l'indemnité." (article 16 de la loi du 30 mars 1898 sur la concession du métro). Cet article de loi tente de prévenir une possible déresponsabilisation de celui qui a pour

16 Cette citation est extraite du rapport de recherche, Mise en auvre et intégration des stratégies de changement à la $\mathrm{R} A T P$, réalisé sous la direction de Michel Crozier. CSO. Paris 1993. Pour l'ensemble des citations de paroles d'agents, afin de ne pas alourdir le texte, nous avons indiqué à sa suite la catégorie de l'agent, l'année de réalisation de l'entretien, les initiales du chercheur responsable de l'étude et enfin en cas de publication, la page dans le texte de référence. 
mandat d'organiser le transport. Il incite "l'entrepreneur à produire la série de règlements adaptant l'article de loi à la réalité du transport, d'interpréter l'impératif de réparation auquel le soumettent les agissements et les pointes de vitesse de ses employés et d'affecter la qualité et la quantité de moyens nécessaires pour parvenir à l'objectif annoncé." [Bucas-Français, 1991 : 83]. 100 ans plus tard, cette controverse reste ouverte.

\section{Le conflit de 1985 : l'amorce d'une inflexion dans la trajectoire du réseau ferré}

Le dénouement de cette crise est ambigu. Dans l'immédiat, en dehors de la suspension de la note concernant les flux résiduels de voyageurs, les conducteurs n'ont obtenu que peu de "choses" : le paiement des heures de grève, des équipements supplémentaires pour favoriser la surveillance de la ligne de portes (installation de bandes blanches en bordure de quais, caméras et miroirs...), l'ouverture de négociation sur la réglementation. Ils ont également "obtenu" que la direction lance une campagne auprès des voyageurs pour obtenir "une certaine discipline". Des "TUC" (travaux d'utilité publique) ont été embauchés sur la ligne A pour éduquer les voyageurs à perdre l'habitude de tenter de monter quand le signal de départ est donné. De l'entrée en station, il n'est pas question. Malgré ce bilan qui peut apparaître maigre, il semble que ce conflit ait eu des répercussions importantes, comme s'il avait été un de ces éléments qui contribuent, par la manière dont ils affectent le groupe conducteur, à modifier la structuration d'ensemble du réseau.

En effet, si dans la succession des faits qui marquent le réseau, aucun lien ne se donne à voir de manière immédiate, certains semblent se relier. Ainsi en va-t-il des grèves de décembre 1986/janvier 1987 déclenchées par le Syndicat Autonome Traction. Non seulement la période de déclenchement du conflit $-22,23$ et 24 décembre reprend de manière planifiée ce qui l'année précédente n'était qu'une coöncidence mais en plus, dans l'espace de la négociation salariale, resurgit la question de la sécurité. Le conflit classique sur les parités salariales propre à la RATP - le dernier échelon des conducteurs doit être équivalent au premier niveau de la maîtrise concours - se résout, pour une partie importante, dans l'obtention d'une prime 
d'incitation à la sécurité pour ceux qui sont classés T3 c'est-àdire au sommet de "1'échelle Traction" ${ }^{17}$.

Ainsi de proche en proche, au travers de l'instance du conflit, se lit la manière dont se trame une histoire. La grève de 1985 restructure les relations entre les éléments disparates du dispositif productif; elle crée de nouveaux rapports entre grève des conducteurs et vacances des voyageurs, entre sécurité et salaire; elle recompose un système de relations professionnelles basé sur la centralité salariale et productive du groupe de conducteurs. Le conflit n'agit pas seulement dans l'espace de la catégorie "en lutte", il modifie l'espace où se déroule la lutte, affectant l'ensemble des êtres qui le peuplent, humains (conducteurs, agents de maîtrise, cadres, voyageurs, politique...) comme non humains (marquage au sol, conduite centrale, réglementation, lois, grille de salaire...).

Cette grève touche à des points essentiels dans le fonctionnement du métro : la responsabilité, la sécurité, les voyageurs, la station; elle marque le dispositif productif dans la définition du travail et du salaire, mais sa résolution passe sous silence ce qui structure les conduites déviantes. En effet tout se passe comme si le conflit qui aboutit à requalifier l'acte du conducteur condamné, avait été l'opérateur d'une transformation du rapport à l'accident. En même temps qu'une sorte de "déresponsabilisation" juridique du conducteur est obtenue - "la faute" est socialisée dans le réseau -, l'acceptation du fait lui-même, "des accidents ou des suicides", semble en devenir la contrepartie naturelle tant pour les conducteurs que pour l'encadrement.

Ce conflit de 1985 nous semble être un des opérateurs majeurs d'une transformation du rapport au suicide, expression générique $\mathrm{du}$ rapport à l'accident voyageur, au sein du réseau ferré. D'une part, par ce qu'il initie un nouveau cycle de conflits qui, à terme, restaure, dans le même temps et de manière contradictoire, le groupe conducteur au centre du dispositif salarial et du processus d'innovation. Tandis que les conducteurs peuvent faire valoir leur prédominance dans les rapports salariaux, le projet AIMT traduit, dans l'espace des techniques, la

17 Ce conflit marque également le terme d'une tentative amorcée par Claude Quin, président de la RATP de 1981 à 1986, de sortir d'un système de relations professionnelles basé sur une économie catégorielle. Ce statu quo ante aboutit au conflit des ouvriers de la maintenance de l'hiver 1988 qui débouche à son tour sur la nomination de Christian Blanc [Foot, 1994 : 6669]. 
focalisation du réseau sur ce groupe pour en restreindre l'importance. Les ingénieurs traitent alors du rapport à l'accident voyageur par défaut quand les conducteurs sont absents. D'autre part, au sein du groupe conducteur, le rapport au "suicide" ne semble plus trouver à s'exprimer sous forme explicite. Tout se passe comme si ce conflit avait contribué à "restructurer" l'idéologie professionnelle du groupe conducteur. C'est cette hypothèse que nous voulons ici examiner au travers de certaines formes d'expression de la mort, de l'accident et de la sécurité.

\section{Une approche du suicide dans des paroles sur le travail}

Dans l'espace de la parole, le premier constat est que "apparemment il y a du non dit mais d'une façon vraiment extrême (...) Il est absolument très rare qu'on puisse parler de "suicide" entre nous d'une autre façon que sur un mode un peu bravache, du genre: "ouais, moi j'en ai eu 3 "" (ancien conducteur et agent de maîtrise, 1996. RF. p.35 ) ${ }^{18}$. Ce silence semble s'être alourdi. Alors que dans notre enquête de 1984, les trente-cinq conducteurs interviewés évoquent explicitement leur appréhension du suicide, dans celle menée entre juillet et novembre 1992 par Élisabeth Chauffier, sur les dix conducteurs interrogés, aucun ne parle spontanément du suicide. La seule évocation se fait dans une réponse brève à une question directe sur ce thème: "personnellement, $j^{\prime} y$ ai souvent pensé (aux quais bondés). Dans les stations où il y a beaucoup de voyageurs, on a toujours peur d'un geste mal venu ou qu'il y ait une personne qui se suicide mais je crois que bon ... on n'y pense pas" (conducteur 1992. EC). Dans son enquête, Elisabeth Chauffier met en évidence que si les conducteurs, dans les entretiens, n'évoquent pas le suicide, ils déclinent leur métier "autour de deux grands axes: la gestion du rapport au voyageur et le rôle des conducteurs à l'égard de la sécurité ferroviaire." (Chauffier, 1995: 32). Entre son évocation "privée", au cours d'un entretien, et son expression publique, par les syndicats conducteurs comme par les responsables du réseau, le fossé se creuse. L'ennui et la monotonie viennent en contre point des rhétoriques valorisant la conduite tandis que la sécurité, la régulation et leur responsabilité sur les voyageurs forment un triptyque qui enserre, sans l'évoquer, le suicide ou l'accident.

La manière dont le suicide parvient, malgré tout, à s'exprimer nous renseigne sur ce qui est en jeu dans le

18 Cette citation est extraite d'un entretien publié [Foot, 1996b]. 
groupe. D'abord, parler de cette peur suppose souvent de ne plus en faire partie: "Toujours être vigilant, c'est horrible, surtout avec le matériel neuf, j'ai peur, car il faut quatre longues secondes pour freiner son métro, quinze mètres avant de s'arrêter" (conducteur inapte, 1991. JFL) ${ }^{19}$. Dans cette situation, si les conducteurs parviennent à contenir en temps ordinaires l'expression du suicide par la "mise en avant", dans leurs discours, de leurs responsabilités sur le "monde derrière", pour reprendre l'expression d'un conducteur, le suicide s'exprime brutalement dans ces occasions où l'institution, par ces injonctions, fragilise les stratégies défensives basées sur la contenance de l'émotion [Dejours, 1993]. Le suicide peut alors surgir par effraction au détour d'une question sur la politique commerciale: "Le voyageur en premier, devant, ça c'est logique mais pas n'importe comment. S'il y a un suicide, le voyageur pas devant, le voyageur s'il veut pas partir, il faut l'envoyer chier... Il faut aller vite. Un suicide c'est pas beau à voir... Un truc comme ça... ou quelqu'un sur la voie. Il n'y a pas de question à se poser. Il faut couper le courant. Si un voyageur traverse la voie, faut pas déléguer. On coupe le courant." (conducteur, 1992. EC) ${ }^{20}$. Dans d'autres occasions, l'introduction de nouvelles règles de travail ouvre des brêches dans les pratiques défensives. L'information voyageur en est une. De temps en temps, l'annonce "suite à incident grave voyageur, le trafic est interrompu..." se transforme en "suite à un suicide, le...".

Parvenir à tenir l'accident voyageur à distance de soi suppose de mobiliser des opérateurs pour chaque registre dans lequel se joue l'expression du suicide. De manière immédiate, quand le conducteur est dans la crainte de l'accident, "le sujet conscient" tend à disparaître pour faire place à un personnage plus flou: "c'est l'habitude du métier qui fait qu'on voit, on voit sans voir d'ailleurs, qu'il y a quelque chose d'anormal. On le sent tout de suite, c'est conscient et inconscient. On voit qu'il y a quelque chose qui ne va pas." (conducteur inapte, 1991. JFL. p.43).

19 Cette citation est extraite d'un entretien réalisé en 1991 pour une étude sur l'inaptitude [Laé, 1991]. J'ai pu retravailler ces entretiens dans une autre perspective grâce à la coopération de Jean-François Laé et Yves BucasFrançais.

20 Cette citation est extraite d'un entretien réalisé en 1992 pour une étude sur les relations professionnelles Gestion du changement et rôle du syndicalisme, Elisabeth Chauffier et Pierre-Eric Tixier, Gip MI. 1997. Ce texte étant une synthèse de différentes monographies, le texte de référence est celui d'Elisabeth Chauffier [Chauffier, 1995]. Le retravail s'est fait sur la base de nombreuse discussions sur les entretiens eux-mêmes avec elle dans le cadre du Gip MI. 
Dans une "fiction", un conducteur raconte ces moments où les corps se livrent, se mettent en jeu dans l'espace de la station - entre les quais, les rails et le train et s'inscrivent dans le travail: "Neuf heures quarante sept minutes et dix secondes, ça n'avance pas. Soudain, ma main se crispe sur le manipulateur de freinage : sur le quai, un jeune beur a fait mine de se jeter sur la voie et me montre maintenant son majeur pointé vers le ciel (...) Les images d'un précédent suicide me hantent quelques instants. Je passe une dizaine de minutes à me débattre avec mes fantômes. Cela me rappelle l'histoire de ce clochard qui est descendu calmement sur la voie, s'est planté face au train qui arrivait et, au dernier moment, sans une hésitation, a fait un bras d'honneur à la vie. C'est une des histoires que l'on se raconte, entre conducteurs, toujours sur un mode humoristique pour conjurer l'horreur. Le mien, il s'est simplement couché en travers des rails. Je me suis crispé sur le frein d'urgence puis j'ai fermé les yeux. Je savais par mes collègues que les images sont dangereuses. Je n'ai pas pu me boucher les oreilles." [Locqueneaux, 1996 : 173-174]. Ne plus voir quand le corps tout entier est engagé dans l'action apparaît une défense bien fragile. Impuissant à éviter le suicide, le conducteur a peu de ressources pour s'en décharger. Ne pas se sentir engagé par cette impuissance à éviter l'accident est d'autant plus illusoire que chaque accident déclenche une enquête policière et que parfois, certains policiers convoquent directement le corps du conducteur dans l'enquête; ils lui demandent de "souffler dans le ballon".

La question de la faute se déplace dans cette convocation du corps. L'enquête objective l'implication du conducteur; elle caractérise son degré de culpabilité. Que cela soit un suicide et, formellement, l'intention du voyageur tend à exonérer le conducteur d'une responsabilité. A contrario, si ce n'est pas la "faute" du voyageur alors celle du conducteur semble être engagée: "Quelques uns ne s'en remettent pas vraiment, surtout lorsqu'il s'agit, non pas de suicides, mais d'accidents. Un gamin qui dérape sur le quai avec ses rollers tout neufs et qui passe dessous, c'est moins facile à assumer que quelqu'un qui se jette délibérément, même si dans un cas comme dans l'autre, on ne peut rien faire pour l'éviter." [Locqueneaux, 1996 : 174].

\section{Campagne de presse, silence et salaire}

Si pour tous, depuis les conducteurs jusqu'aux cadres, l'usage du terme de suicide est l'opérateur d'une mise à distance de l'accident et de la mort, la distinction entre accident et suicide est, pour les conducteurs, une ligne 
de partage ténue. Cela ne contient pas vraiment la faute; ce n'est qu'“à la limite (que) ce n'est pas de sa faute" (conducteur inapte, 1991. JFL). Si l'usage du mot suicide autorise, en apparence, à renvoyer la mort, les blessures et la souffrance du côté des voyageurs, la prise en charge du traumatisme semble alors être du seul ressort des conducteurs. Pourtant, l'institution aussi en parle. Elle en fait même un élément constitutif du métier: "Dans le stage (de formation à la conduite) on nous disait souvent: "si vous avez un incident ou un suicide par exemple". Cela m'a choqué." (ancien conducteur et agent de maîtrise,1996, RF. p.29).

Cette prise en charge institutionnelle s'élabore se traduit in fine dans le salaire. Là se paie le silence: "J'ai refusé d'entrer dans cette logique enfin pas vraiment sinon je serais parti. Je ne l'ai pas refusé à ce point, mais je ne l'ai pas totalement acceptée. Je me suis dit: "si l'un des aspects du métier c'est d'avoir à tuer quelqu'un - c'est vraiment comme cela que je l'ai ressenti - je ne veux pas rester là dedans " (...) J'ai eu un problème qui peut expliquer ma difficulté à analyser ces phénomènes (de résistance à l'expression d'émotions lors d'un suicide) qui peut expliquer que je n'ai pas tellement envie de jeter un regard sur ça dans la mesure où ça a failli m'arriver aussi. J'ai accepté de conduire aussi. J'y pensais souvent, mais pas assez pour quitter la RATP." (ancien conducteur et agent de maîtrise, 1996. RF. pp.29 et 35). Avant même que l'agencement "matériel" du dispositif de conduite ne contraigne les actes de travail, c'est d'abord l'instauration d'un rapport de subordination accepté qui amène l'agent à soumettre la régulation de sa peur à la régulation des trains, à accepter de faire "dominer" la peur produite dans l'agencement du travail de conduite par "son" respect des normes de production et de qualité de service instituées sinon "la conscience aiguë (...) du risque d'accident, obligerait l'ouvrier à prendre tant de précautions individuelles qu'il deviendrait inefficace au plan de la productivité" [Dejours, 1993 : 89].

Accepter le déni de la réalité du travail par l'institution, que traduit l'agencement de la conduite, c'est rentrer aussi pour soi dans une dénégation de son travail : "Le suicide est quand même une certaine hantise. On apprend vite. On n'a pas la fonction et puis l'idée en tête lorsqu'on est à la RATP, conduisant un train, de faire ça pour tuer, hein, sinon on est mercenaire ou on est à... Il y a d'autres endroits où on est sûr de s'offrir un cadavre quand on veut." (conducteur inapte. 1991. JFL). 
Si le lien n'est pas explicitement fait entre "accident voyageur" et salaire, c'est pourtant une prime d'incitation à la sécurité qui, lors de la grève de 1986, restaure la position centrale du groupe conducteur dans la grille salariale. C'est de nouveau autour du salaire et de la sécurité que se noue le conflit de novembre 1992 qui débouche sur le départ de Christian Blanc. Un des faits remarquables de ce conflit est en effet la manière dont le salaire a été instrumenté par la direction pour isoler les conducteurs. Les journalistes remarquent cette stratégie de la direction : "Publier les salaires de ses propres agents est assez inhabituel de la part d'un patron public" (Le Fig-Eco du 16/4/92). Cette "mise sous presse" des conducteurs sera réactivée en novembre. Les conducteurs récusent cette stratégie de dénonciation que ce soit au cours d'entretiens " $A$ partir du moment où on donne les conditions de travail, il faut donner tous les éléments... Quand on balance ça dans le public on paraît être une catégorie ultra favorisée. C'est inadmissible d'entendre ça dans les médias, c'est choquant." (conducteur, 1992. EC. p.35) ou par le biais syndical "est-ce responsable de livrer en pâture aux journalistes avides de sensationnel le montant des salaires gonflés et fausses informations tels que le temps de travail réduit?" (SAT, $28 / 3 / 93)$. Face à cette campagne, ils restent pourtant sans voix pour dire l'autre face du salaire, pour dire le travail de la peur contenue dans le salaire: "(Le travail) ne se résume pas à être un simple lampiste qui se trouve dans l'aquarium et qui appuie sur un bouton. La responsabilité est plus grande qu'on ne le croit. Certaines personnes ont tendance à minimiser notre rôle, on est des presse-bouton, on est trop payé." (conducteur, 1992. EC. p.35).

Ils se sentent "piégés", mis dans l'impossibilité de dire leur travail avec ces "fantômes" qui les habitent: "Des fois, on les revoit. C'est malgré moi, il y a des fois... la nuit... même encore maintenant que je ne suis plus sur les trains, des fois, j'y pense comme ça, c'est normal, on a toujours un... c'est le subconscient qui travaille. Et des fois je les revois. Ma femme me dit: "t'as fait un cauchemar cette nuit". Déjà je me réveille, je suis tout en nage. (...) Se voir dans l'impossibilité de pouvoir faire quelque chose, je crois qu'il arrive un moment où vous ne pouvez plus le supporter. De savoir qu'on a tout dans les mains pour faire quelque chose et en même temps être dans l'impossibilité de pouvoir le faire. Là il y a des choses, qu'on soit à jeun, qu'on soit bourré, y a des choses qu'on voit qu'on fait... mais là on est vraiment impuissant, vraiment impuissant parce que c'est fait." (conducteur inapte, 1991. JFL).

Tout se passe comme si dire la tension des heures de pointe où il "faut faire attention aux gens qui sont sur les quais, 
parce qu'il y a les suicides, même les accidents (...) (où il) faut quand même avoir une attention soutenue pour voir si y en pas un qui tombe." (conducteur inapte, 1991. JFL) revient à se placer dans une contrainte insoutenable où si les conducteurs disent leur peur, s'écroule alors la stratégie défensive du groupe, basée jusque là sur son refoulement : "est-ce que cela veut dire que l'on doive occulter complètement l'action pour pouvoir reconduire un jour, et si possible, dès le lendemain? Est-ce que, sinon, si on commence à en parler, il devient impossible de conduire après?" (ancien conducteur et agent de maîtrise, 1996. RF. p.35). Sommés par leur direction, face au public, de reconnaître qu'ils sont bien payés et ont de bonnes conditions de travail, les conducteurs ne peuvent pas dire leur peur du voyageur sans faire s'écrouler leurs défenses. Alors, ils dénoncent le procédé sans pouvoir dire ce qui en fait pour eux l'infamie.

Ce n'est que de manière détournée qu'ils disent $q^{\prime}$ 'une de leur fonction est d'assumer le suicide et l'accident: "Tant qu'on peut démontrer qu'on est utile, sur les avaries, pour les dépannages, pour plein de choses, pour évacuer les gens lors des suicides ou d'incidents en ligne... tous les thèmes qui peuvent montrer qu'on est utile pour éviter une certaine déqualification." (conducteur, 1992. EC. p.35).

\section{Taire la peur et contenir l'émotion : une pratique défensive soutenable?}

Sans le couvert des mots, la peur trouve d'autres voies pour s'exprimer. Si on ne peut, par définition, entendre un silence, les processus de refoulement de ce sur quoi porte la peur laissent par contre des traces. L'observation du silence passe alors par celui de son travail sur les groupes et dans la mise en forme des agencements productifs. Le silence fait sur la peur du voyageur acquiert dès lors le même statut que l'inconscient chez Devereux: "Le fait que j'observai la pause du patient ne signifie pas que j'ai observé directement son inconscient. Cette pause correspond seulement aux empreintes de pas laissés par un fugitif traqué, empreintes qui peuvent fournir des informations sur le fugitif." [Devereux, 1980 : 405].

L'importance qu'un chercheur accorde à une "chose absente" dans les théories et discours indigènes doit donc être soumise à l'épreuve de ces empreintes. Ces portes palières qui matérialisent une réponse concrète donnée par un groupe, celui des responsables, à la crainte d'un accident voyageur, constitue déjà une "trace" de son importance 
pour le réseau. La grève de 1985 en est une autre. Mais d'autres marqueurs existent. Certains affectent directement les productions de discours. Par exemple, au début des années 80 , la RATP régule, avec les journalistes, la diffusion d'informations sur "le poussage". Ce phénomène disparaîtra, si ce n'est du métro, de la Une des journaux. Mais vouloir rendre invisible peut aussi conduire à ne plus «voir» les images que l'on produit. Ainsi la direction publiera un dessin humoristique dans sa brochure «Le progrès partagé », nouveau contrat social lancé par Christian Blanc en 1992, dans lequel un agent se jette par la fenêtre, depuis son bureau, sur la voie, devant un train sans comprendre que ce dessin représente potentiellement un suicide: "La question de ce dessin? personne ne s'en est rendu compte. Si on avait pu l'interpréter, on ne l'aurait pas mis. Si on l'avait vu, c'est sûr qu'on ne l'aurait pas pris" (entretien avec un responsable de la communication interne. 1997). À $l^{\prime}$ inverse, au sein de l'organisation, $\mathrm{d}^{\prime}$ autres images sont produites pour montrer sans dire.

\section{Montrer la mort, canaliser la peur}

Dans certains terminus, "les conducteurs avaient affiché dans le local de départ un tableau d'honneur avec, en regard de chaque nom de conducteur, des petites têtes de mort représentant chacune un suicide." [Locqueneaux, 1996 : 174] ou les jours : "au départ, il y a un calendrier. L'un des chefs de départ met une tête de mort sur le calendrier à chaque fois qu'il y a un suicide." (ancien conducteur et agent de maîtrise, 1996. RF. p.39). Sans un mot car "c'est là et personne ne dit rien" (idem), dans l'espace principal de socialisation de la ligne, "le local de départ" en terminus, les têtes de mort dessinent une manière de conjurer la peur. Des jours sont mauvais, des conducteurs attirent le suicide: "Certains conducteurs, peu nombreux, partent à la retraite sans avoir eu de suicide. D'autres détiennent des records impressionnants (...) Les deux premiers de la liste avaient respectivement 8 et 7 suicides à leur actif. Et le premier n'avait que dix ans de conduite." [Locqueneaux, 1996 : 174]. Certains conducteurs cumulent les deux facteurs et activent l'imaginaire du groupe. Des histoires circulent sur "ce" conducteur qui a eu un suicide dès son premier jour ou sur cet autre qui cumule plusieurs suicides dans son service: “On raconte l'histoire d'un conducteur qui en a eu un au début de service, puis qui après avoir sacrifié aux formalité d'usage, est remonté sur son train pour finir son service et s'en est "farci" un autre" [Ibid.].

Par la création de cet espace graphique, des agents - un chef de départ dans un cas, des conducteurs dans 
l'autre - agissent pour révéler cet objet d'appréhension sans toutefois le verbaliser. Dans ce processus de représentation des "suicides", intermédiaire entre le dit et le non dit, un paradoxe émerge, celle de la surestimation du nombre et de "l'efficacité" des suicides. Par la médiation du dessin de la tête de mort, en effet, tous les accidents d'exploitation mettant en jeu une rame, la voie et un voyageur tendent à acquérir un même statut de suicide. Ainsi, progressivement la diversité des causes d'accident s'efface au profit de la seule qui engage la "faute" du voyageur, le suicide donc. De plus, c'est l'accident qui est signalé et pourtant c'est la mort qui est dessinée. Dans cette traduction graphique, l'efficacité morbide de l'accident est surreprésentée: "J'estime à 50 pour cent ceux qui en meurent sur le coup. Pour les autres, soit ils meurent à l'hôpital, soit ils restent infirmes toute leur vie. Mais il faut noter que quelques uns s'en tirent miraculeusement." [Ibid.]. En fait, le taux de "réussite" des tentatives de suicide proprement dits est sensiblement plus faible; suivant les années, il varie entre moins de $20 \%$ et un tiers. Pour les autres accidents mettant en jeu la voie et/ou les rames, le taux est beaucoup plus faible, inférieur en général à $10 \%$. Ces décalages permettent de comprendre que ces dessins ne représentent pas la mort du voyageur mais la réalité de la peur du conducteur, de sa potentialité de mise à mort du voyageur. Ces dessins tentent, a posteriori de chaque accident, de capter cette peur de tuer qui flotte dans le groupe pour le fixer sur des conducteurs et/ou des jours.

Ce travail d'attribution s'apparente à une tentative de conjurer la peur de tous par la production d'un tableau où le risque apparaît pour n'être pas égal pour tous. Certains attirent plus que d'autres le voyageur. Dans cette procédure d'imputation aux conducteurs d'une capacité différentielle d'attraction des voyageurs, d'une capacité à "tenter" le suicide pourrait-on dire, le groupe désigne ceux qui n'ont pas de chance. Ainsi, par cette forme graphique, "l'usage défensif de la technique du bouc émissaire peut fort bien s'enseigner" [Devereux, $1970: 9]$ et se transmettre. Certains conducteurs deviennent ces sortes de boucs émissaires qui, chargés de la peur du groupe, auraient pour fonction d'en attraper la cause. Ils n'en sont pas pour autant responsables des accidents; ils ne sont pas coupables de la malchance qui s'attache à leur personne même si elle pèse sur eux. Dans les échanges de service entre conducteurs, cette innocence de la personne se perçoit. Qu'il arrive un suicide pendant qu'un conducteur en remplace un autre et tout de suite se met en place un processus de transfert de la faute soit que le service était celui d'un conducteur "attracteur" de suicide soit que 
le conducteur remplaçant l'était lui-même. Cette procédure d'attribution d'une qualité que ne possède pas la personne mais qui la possède et la pousse à l'accident n'est pas le propre de groupes ouvriers. Elle nous semble équivalente à celle qui conduit un médecin du travail à caractériser un ouvrier comme "polyaccidenté prédisposé" c'est-à-dire appartenant à un groupe, à "une minorité de 1 à $2 \%$ subissant un nombre important d'accidents graves (...) le prédisposé ne l'est jamais par hasard". Cette caractérisation s'est faite a posteriori d'un accident plus grave: "Il a suffit de muter l'ouvrier pour que baisse son "accidentabilité ", ce qui revient à dire que ce qui a changé dans la situation ce sont bien les conditions de travail et non le travailleur." [Dejours, 1993 : 144145]. Dans ce processus de désignation d'une prédisposition des personnes à l'accident, sont abolis à la fois le hasard et les conditions de travail. La psyché du sujet est seule, par cette procédure, à être en question.

\section{Des conduites dangereuses et de défi au "bon sens"}

Les pratiques de travail du groupe conducteur manifestent leurs formes d'action pour dominer la peur. La première technique consiste en l'apprentissage de l'entrée en station: "Tu testes, tu essaies de rentrer de plus en plus vite en station pour voir si tu vas quand même t'arrêter au bout." (ancien conducteur et agent de maîtrise, 1996. RF. p.30). La peur du suicide ne résume pas le travail de conduite. Un conducteur doit avant tout, du point de vue de la hiérarchie, maîtriser les arrêts en station et respecter la régulation d'intervalle. Cela doit se traduire dans une maîtrise de l'arrêt en station d'une rame arrivant à vitesse élevée. Que la rame entre à trop faible allure ou que, au moment de l'arrêt, elle soit "engagée sous tunnel", et c'est la manifestation d'un manque de professionnalisme. Une fois cet apprentissage réalisé, le travail semble tourner à la routine.

Pourtant des pratiques, collectives cette fois-ci, d'entrée dans certaines stations ne peuvent se réduire à cette fonction d'apprentissage: "Sur une ligne où j'ai conduit, équipée en matériel pneu, à une station précise, le défi entre conducteurs consistait à flirter avec la vitesse limite." (ancien conducteur et agent de maîtrise, 1996. RF. p.30). Ce comportement des conducteurs "flirte" avec un ensemble d'éléments qui, d'ordinaire, structure pour partie, sur le mode de la retenue et de l'interdit, l'identité professionnelle: la peur, le respect des vitesses et des distances. Là, au contraire, ils en rajoutent. Nous sommes 
dans une situation "classique" pour la psychodynamique du travail où "conjurer le risque exige (...) que les ouvriers ajoutent parfois au risque du travail celui de performances personnelles et de véritables concours d'habileté et de bravoure." [Dejours, 1993: 89]. Freiner le plus tard possible dans la station, tenir le plus longtemps possible la vitesse limite, tout en s'arrêtant au bon endroit, et alors le conducteur devient un "virtuose" de la conduite ou plus précisément de l'entrée en station. Sur les lignes à roulement pneumatique, certaines stations se transforment donc en "arènes des habiletés techniques: lieux d'épreuves des capacités de l'individu confronté dans ses actes au fonctionnement des objets techniques et placé au carrefour des jugements d'autrui" [Dodier, 1995 : 220]. L'encadrement est convoqué implicitement pour arbitrer leur concours. Le dépassement d'une vitesse limite oblige la hiérarchie à sanctionner le conducteur. Cette sanction devient alors une sorte de reconnaissance officielle de la virtuosité du conducteur. Cette inversion de valeur attachée à la sanction entre le groupe conducteur et la maitrise constitue un indice du clivage sur le sens du travail au sein de la communauté ferroviaire. Le défi à la sécurité se double d'un défi à la hiérarchie.

L'existence de ce type de défi, par les formes sociales $q^{\prime}$ 'il prend, indique que nous nous trouvons dans une situation où le "traitement" de la peur ne procède pas d'un mode idiosyncrasique de défense mais d'un mode culturalisé au sens où la culture est "une manière structurée d'appréhender la réalité tant sociale qu'extra-sociale" [Devereux, 1970 : 83] c'est-à-dire qu'elle "est le système qui non seulement rend cohérent l'espace social, mais aussi, et surtout, le système intérieur des individus qui leur permet de clôturer leur espace psychique" [Nathan, 1994: 175-176]. Cette pratique manifeste que les régularités constatées, tant dans les pratiques ordinaires de travail ou dans les formes de retenue de l'émotion, que dans les pratiques langagières ou graphiques, ne sont pas seulement un artefact sociologique projeté dans la réalité sociale. Elle atteste que pour devenir conducteur, il faut certes satisfaire au concours officiel mais aussi à d'autres épreuves sociales où les conduites sont également formées et jugées.

Les différents processus de sélection, officiels ou non, ne garantissent pas pour autant que chaque conducteur résiste à sa mise à l'épreuve quotidienne dans la production. Certains "craquent". Cette culture qui, "quoique d'origine externe à l'individu", leur permettait "de se mouvoir, de penser, d'aimer et de travailler en évitant frayeur et perplexité" [Nathan, 1994: 179] devient inopérante. A suivre, le 
processus par lequel l'un d'entre eux tente d'échapper à l'emprise de l'espace productif, nous avons l'impression de pouvoir quasiment toucher du doigt tous les éléments qui sont liés entre eux pour faire que cela tienne dans les temps ordinaires: "En heure creuse, il n'a pas marqué l'arrêt à une station et il a loupé son freinage à la station suivante, engageant une partie de son train sous tunnel. Il nous a dit que c'était le pilotage automatique qui ne fonctionnait pas. La bande enregistreuse indiquait qu'il était en conduite manuelle, mais il maintenait sa déclaration. Après enquête, on s'est aperçu que le tapis du Pilotage Automatique était démonté depuis 3 mois. Pourtant, cela ne l'a pas empêché de maintenir son explication." (ancien conducteur et agent de maîtrise, 1996, RF : 38). Les symptômes se nouent précisément dans le moment de la production où se structure, pour une part importante, la culture du métier - l'entrée et l'arrêt en station - et dans le lieu où les pratiques de travail sont mises à l'épreuve d'un conflit entre le fait d'être agent de sa conduite avec la conduite manuelle contrôlée ou d'en être le patient avec le pilotage automatique. Sur un mode en apparence pathologique, il se défend d'être responsable de "la" faute en refusant non plus sa peur mais la réalité du monde : "On a pris la décision de le descendre du train parce que tu ne laisses pas conduire un type dans cet état. Ce qu'il avait fait était déjà très limite, mais en plus il soutenait des choses invraisemblables. Il disait: "on m'accuse parce qu'on veut couvrir les trains qui sont déficients" (ancien conducteur et agent de maîtrise, 1996, RF : 38).

Ce processus de marginalisation d'un individu s'apparente à ce que l'on retrouve dans d'autres espaces professionnels - "Si un ouvrier ne parvient pas à reprendre l'idéologie défensive du bâtiment à son propre compte, s'il n'arrive pas par ce moyen à surmonter son appréhension, il devra cesser le travail. Le groupe (...) élimine celui qui ne supporte pas le risque" [Dejours, 1993: ] - en même temps qu'il prend un tour plus institutionnel par la procédure instaurée pour juger de l'aptitude ou non d'un salarié à occuper son "emploi statutaire". Du fait de l'existence d'une garantie d'emploi pour ceux qui deviennent "inaptes à leur emploi statutaire", les scrupules à "descendre" quelqu'un du train en cas de problèmes, à l'éliminer du groupe, sont amoindries : "On peut prendre ce genre de décision sans trop de problème de conscience parce qu'on sait qu'il conserve sa paye et son statut. Il y a toute une procédure tant sur le matériel que sur la personne pour déterminer les conséquences à tirer d'un incident." (ancien conducteur et agent de maîtrise, 1996, RF : 38). 
Dans les interactions au sein du groupe pour que le déviant recouvre son aptitude à la conduite, ce dernier focalise ce qui dérange: "Dans ce cadre, plus tard, on l'a convoqué, un après midi, à la visite médicale pour juger de son inaptitude éventuelle. Durant la période où il n'avait pas le droit de conduire, on s'était arrangé pour mettre tous ses services en matin. "Je n'irai pas à la visite parce que c'est à 14 h et moi je travaille le matin". Alors, je l'ai convoqué "Ecoute à mon avis tu es mal parti, fais au moins preuve de bonne volonté. On t'a arrangé tous tes changements de services, tu passes tes journées assis à attendre. Là, tu as une chance de remonter sur le train, joue-la à fond. Ne reporte pas cette visite sous prétexte que tu travaillais ce matin. Montre ta motivation ". J'ai essayé de lui expliquer; je lui ai rappelé l'enquête. A la fin il m'a dit. "Je sais pourquoi tu me dis ça, t'es pareil que les autres ". Je lui dis "Oui sûrement, oui, mais comment sont-ils, les autres?" "Vous êtes tous jaloux parce que je reste assis sur ma chaise au lieu d'aller travailler!" (ancien conducteur et agent de maittrise, 1996, $\mathrm{RF}:$ 38-39).

Le déviant ne parvient pas à produire, pour les autres, le sens de ses actes ${ }^{21}$. Peut-être faut-il alors remonter à ce qui s'est désaccordé dans le monde pour comprendre le désordre qui s'est saisi du conducteur. Le démontage du tapis du pilotage automatique est peut-être ce qui ouvre une brèche dans l'équilibre entre ses mondes intérieurs et extérieurs. Par cette panne du PA, le conducteur a perdu également sa liberté d'être responsable ou non de ses actes. Il ne peut plus reporter la responsabilité des entrées en station sur l'automate. Par cette défaillance des objets techniques, il est conduit à devoir éventuellement assumer seul la responsabilité d'un accident. Cela peut suffire pour rendre le travail insupportable et que rester assis à ne rien faire soit le travail dorénavant supportable.

Seul, ce conducteur devient l'idiot c'est-à-dire l'étranger au métier. La culture de métier n'est plus une

\footnotetext{
21 Ce cas nous semble exemplaire d'un processus d'aliénation mentale où "l'individu est coupé à la fois du réel et d'autrui" [Sigaut, 1990:170] dans le cadre d'un processus d'aliénation sociale du groupe conducteur du fait du déni de son rapport à la réalité par autrui. Autrui est défini par l'ensemble de ceux qui participent du déni de cette réalité c'est-à-dire que les conducteurs participent aussi de ce processus. La proposition de François Sigaut qu'autour des trois termes EGO_REEL-AUTRUI puissent se produire trois types de ruptures correspondant à trois types d'aliénation (mentale : Ego se coupe du rapport Réel-Autrui; sociale : le rapport Ego-Réel se coupe d'Autrui ; culturelle : le rapport Ego-Autrui se coupe du Réel) nous semble particulièrement féconde pour analyser la dynamique culturelle du réseau ferré.
} 
ressource pour lui. Il ne parvient plus à se servir de "la chambre froide culturelle" inventée par le groupe conducteurs pour y "reléguer ses fantasmes", pour y "entreposer ses conflits intérieurs". Il n'arrive plus à "leur donner une expression abstraite et générale en les insérant dans le corpus impersonnel de la culture" et, par là, "à les retirer de la circulation "privée", donc idiosyncrasique" [Devereux, $1970:$ 12]. Dans cet espace du métro, "la chambre froide culturelle" des conducteurs ne relève pas principalement d'un mythe constitué dans la parole mais d'une construction hétérogène où s'agrègent les conduites de retenue de l'émotion, le silence sur la peur, les objets traçants les responsabilités - la CMC ou le PA - les pratiques de défis, les dessins, le salaire ... Cette "chambre froide" est une construction instable car sans cesse un accident vient rappeler qu'elle n'est pas efficace dans la réalité du voyageur. Cela suppose que, tout le temps, de nouvelles pratiques viennent colmater les fissures qui se forment dans ces "chambres froides" sinon, à l'instar de l'idiot, le groupe conducteur menace de s'arrêter. Dans cette dynamique, c'est le groupe tout entier cette fois-ci qui est amené à se singulariser, à se constituer non pas dans un idiolecte, puisqu'il faut faire silence, mais dans une "idiopraxis" et à devenir ainsi de plus en plus étranger aux autres mondes.

\section{Quel sens aura météor pour les conducteurs?}

Dans cette analyse du métro nous avons tenté de rompre avec une stratégie de recherche qui, en suivant par trop les acteurs, redouble de fait la division du travail et enferme la théorie de chaque groupe dans son cadre organisationnel. Nous avons parié sur les continuités qui travaillent l'organisation pour comprendre la dynamique de transformation du réseau métro. Le projet Météor constitue un point de départ, car, déjà, au travers de sa définition comme ligne "entièrement automatique", ses inventeurs et responsables établissent un lien entre l'automatisation et le travail des conducteurs. Mais d'emblée ce lien apparaît étrange, constitué en creux du sens des mots dans le plein des objets techniques, comme s'ils peuvent, eux, se permettre d'être indifférents au sens. Ces objets viennent alors trancher les zones de flou de l'organisation et rendre compte, à leur manière, des lacunes des discours.

En particulier, les portes palières, en clôturant l'espace entre le quai et la voie, nous ont permis d'interroger l'avenir du réseau et le travail des conducteurs. 
A partir de leur enracinement sur les quais, nous avons essayé de cerner comment les responsables du réseau et les conducteurs appréhendent le voyageur. Par la confrontation, dans l'espace de ce texte, d'une technique potentielle, celle de Météor, avec celle à l'œuvre actuellement sur le réseau, la peur de l'accident voyageur nous est apparue comme un élément structurant de ces deux groupes.

Les responsables du réseau sont dans un temps différé par rapport au transport des voyageurs. Leur confrontation au voyageur passe par de nombreuses médiations: ligne hiérarchique, services fonctionnels, réglementation, définition des objets techniques ... Leur peur est à la fois plus distante mais aussi plus structurée par l'existence d'une idéologie sécuritaire qui se manifeste dans des procédures formalisées de prise de décision et de cahiers de charges. Leur travail se fait dans la crainte de l'accident voyageur, comme on dit de quelqu'un qu'il vit dans la crainte de Dieu. Il existe des codes et des règles qui permettent à chacun de s'approprier dans son travail les principes de précaution du milieu y compris les manières de se protéger des conséquences d'une incertitude. La crainte, loin d'inhiber l'action, l'oriente vers des stratégies de prudence efficace.

Les conducteurs sont dans le temps réel du transport $\mathrm{du}$ voyageur. Leur expérience du voyageur est directe et se réalise dans la répétition des situations où sa présence se manifeste sous des modalités variées: une foule dense sur un quai étroit, des jeunes jouent à se (lui) faire peur, une jolie fille semble l'attendre, un ivrogne titube, un type se jette sous la rame ... Leur peur est immédiate et s'intègre donc dans leur manière d'être. Ils n'ont quasiment pas de ressources pour calmer leur peur, ni la mise en pratique de savoir-faire de prudence, ni le secours des objets. Leur travail se fait dans la frayeur, dans une peur qui se déploie au milieu du fracas du train et du voyageur. Il n'existe pas de moyens re-connus pour prévenir l'accident, ralentir n'en est pas un. La frayeur, loin d'induire des stratégies de prudence, s'exprime alors dans l'exacerbation de la peur et la contention de l'émotion.

Ainsi cet espace frontière entre le quai et la voie, nous a permis d'explorer deux instances du réseau, celui des responsables et des concepteurs et celui des conducteurs, de voir qu'ils ont en commun la peur du voyageur. La peur se mêle des pratiques, trame les techniques et contribue aux salaires. Ces agencements, dès lors, ne se laissent pas 
réduire à être perçus sur le seul registre de la fermeture de l'espace dans lesquels chaque groupe travaillerait avec ses propres outils. D'emblée, ils supposent d'être réfléchis dans leur manière de s'ouvrir aux voyageurs, au public. Il faut penser les "portes" [Simmel, 1988 : 164] qui permettent au travail localisé d'accomplir sa mission générale. Elles raccordent de manière particulière un espace limité, celui de la production, à un espace illimité, celui de la consommation. Parce que l'agencement organisationnel spécifie ce qui est interne à l'espace salarial de ce qui lui est externe, sa définition relève de la compétence des responsables. Par là, il est aussi un "pont" qui relie des espaces finis de l'entreprise [Id. 164-165], ceux par exemple de la conception et de la décision avec ceux de la production. Les éléments qui agencent le travail à la production ont donc toujours le double statut de porte vers le public et de pont entre groupes salariés. Les formes de clôture et d'ouverture de ces portes lient indissociablement le voyageur, le conducteur et l'ingénieur.

Objets complexes, ceux qui cadrent le travail, ne découpent pas seulement l'espace industriel pour focaliser les interactions productives, mais ils autorisent aussi à globaliser "activement des interactions successives par un ensemble d'instruments, d'outillages, de comptes, de calculs, de compilateurs qui permettent de passer d'une relation compliquée, enfin isolable, à d'autres relations compliquées, enfin reliées" [Latour, 1994: 594] ; ce sont eux qui permettent de passer d'une "socialité complexe à une socialité compliquée." [idem]. Dépositaire d'une part importante de la complexité de nos univers productifs, ces objets sont des médiateurs entre instances du monde industriel; ils permettent donc $\mathrm{d}$ 'analyser les processus d'entredéfinition des acteurs car "un dispositif technique n'est jamais aussi muet qu'on pourrait le croire. Cette traduction, qui fait passer un groupement de non humains du silence à la parole, s'opère en de multiples occasions." [Callon, 1991 : 200].

Faire parler ces objets complexes suppose que le chercheur prenne quelques précautions et quelques risques.

Dans l'ordre des précautions, la démonstration procède de la multiplication des traces empiriques, du croisement des discours et du changement des registres dans l'analyse. L'hypothèse, en l'occurrence celle de l'importance de la peur et de son refoulement, n'est plus suspendue à l'arbitraire d'un énoncé théorique, à l'autorité du savant ou à une simple série linéaire d'associations. Au contraire, l'hypothèse prend alors corps dans la 
convergence des théories de chacune des séries d'associations convoquées dans la démonstration. Les objets du terrain viennent stabiliser la théorie.

Dans l'ordre des risques, c'est d'abord celui du terrain, de ce que l'on y voit et de ce qu'on en rapporte. Là, par définition, pour peu que l'on se laisse aller dans l'empirique, le terrain avec ses objets et ses acteurs nous obligent à les penser, à les mettre en mots et que ces mots résistent à leur épreuve, à leur expérience. Dans ce cadre, poser une hypothèse, c'est s'exposer à son tour. C'est rétablir un peu de symétrie entre le chercheur et son terrain.

Voilà en fait le but de ce texte. Ecrire un nouveau rapport entre deux instances - conception et production où des groupes semblent à la fois poursuivre des histoires parallèles et se construire de plus en plus en symétrie. Réfléchir à ce réseau ferré dont la dynamique gravite autour de cette place du conducteur qui en polarise les rapports internes. Les uns tentent sans cesse de restaurer leur place nodale dans les rapports salariaux par les conflits tandis que les autres tentent de les contourner, voire de les supprimer par les réformes organisationnelles et les processus d'automatisation. Cet antagonisme semble irréductible tant à chaque moment "le conflit n'est pas seulement l'affaire des parties, mais du groupe dans son ensemble, (...) chaque partie lutte pour ainsi dire au nom de celui-ci." [Simmel, 1992 : 66]. Dans cette dialectique brutale où aucun des acteurs ne parvient malgré tout à éliminer l'autre, les traces des conflits, en particulier celui de 1985, sont autant de cicatrices qui viennent souder encore plus étroitement leur communauté irrémédiable. Dès lors "l'accident ou le suicide" devient une fatalité contre laquelle, pour l'instant, nul ne peut rien. Après ce moment la peur s'est distribuée sous de nouvelles modalités dans les agencements organisationnels.

Chaque groupe dans ses différentes techniques a mis au point de nouvelles "chambres froides culturelles" pour traiter sa peur. Parallèlement aux formes habituelles de précaution en usage dans le réseau actuel, les responsables, avec Météor, se sont dotés de portes palières afin que la peur n'ait pas lieu d'être, en tout cas pas celle liée à l'intrusion de voyageurs sur la voie. L'existence de cette clôture physique du tunnel, par son efficacité pour le voyageur, rend inutile de se construire une défense idéologique spécifique. Par contre, pour les conducteurs, leur confrontation à cette nouvelle technique, se fera à chaque accident ou suicide. A chaque fois, cela rappellera 
au groupe conducteur que les automates, à l'instar des responsables, comme leur double, n'ont pas, eux, à devoir inventer une "idéologie défensive de métier". Ils sont, grâce aux portes palières, plus efficaces pour ce type $\mathrm{d}$ 'accident. Les conducteurs devront alors tout le temps renforcer leur efficacité non pas sur le terrain de la technique mais en "travaillant" leur imaginaire afin de se défendre contre leur impuissance à agir en sécurité pour le voyageur et de ne pas être menacés dans leur intégrité psychique. Jusqu'au moment où leur stratégie défensive, pour faire face à leurs "frayeurs" des entrées en station, sera certainement insoutenable. Alors le groupe s'arrêtera-t-il ? La sortie du conflit pourra-t-elle être un recours accru à l'automatisation?

Comment sortir de cette dialectique archaïque où les actes techniques, qu'ils se produisent sous forme d'objet technique ou de mouvements du travail, se répondent sans pouvoir faire sens? Autrement dit, la question est de savoir sur quel mode Météor fera événement dans le réseau. Avec lui, l'histoire du métro s'écrira-t-elle toujours de manière "automatique" c'est-à-dire inconsciente, ou Météor permettra-t-il qu'elle se fasse par une autre prise de sens sur la réalité du travail?

"L'automate et ses portes palières" ou "le conducteur et ses peurs", voilà la seule alternative qui semble être posée. Pourtant, n'y a-t-il pas dans toutes ces histoires autour de la conduite, matière à trouver des "objets" qui permettent de "refroidir", de "domestiquer" la peur présente de manière diffuse dans le réseau. Produire un nouvel agencement de conduite, qui intègre non plus une peur "sauvage" mais une peur "civilisée", suppose d'inventer des "chambres froides culturelles" qui permettent à la peur de devenir étrangère au groupe, d'être à son tour aliénée. Ces "objets", pour qu'ils soient des "êtres moraux", ne peuvent alors se passer de "théories indigènes" qui, en leur donnant sens, les chargent d'une force qui aide à supporter ces univers [Nathan, 1991, 1993 : 40-46].

Robin Foot 


\section{Bibliographie}

Akrich, M. (1987), Comment décrire les objets techniques. Technique et culture. 9, 48-64.

Bouvier, P. (1978). Métropolis. Paris : Tep/Alternatives.

Bouvier, P. (1985). Technologie, travail, transport. Paris : Librairie des Méridiens.

Bucas-Français, Y. (1991). Logique de la concession et généalogie de la notion d'aptitude. In I. Joseph, JF. Laé, D. Bonniel, Y. Bucas-Français. Généalogie et itinéraires de l'inaptitude. Paris : Réseau 2000/RATP. pp.75-94.

Callon, M. (1991). Réseaux technico-économiques et irréversibilités. In R. Boyer, B. Chavance, O. Godard, (Ed). Les figures de l'irréversibilité en économie, Paris : EHESS. pp.195-230.

Chauffier, E. (1995). Construction des identités collectives, des compétences et ambiance de travail, une ligne de métro à l'épreuve de la “ modernisation ". Mémoire de DEA de sociologie. Université de Nanterre.

Crozier, M. (sous la direction de). Drevon, Gatto, Rothier Bautzer. (1993). Mise en xuvre et intégration des stratégies de changement à la RATP. Rapport de recherche. Paris : CSO.

Clot, Y., Rochex, JY., Schwartz, Y. (1990). Les caprices du flux. Marseille : Matrice.

Cru, D. (1985). Les tailleurs de pierre, langue de métier et organisation du travail. Travail. 7, 48-55.

Cru, D. (1997). La paradoxale éloquence sur la peur des agents de conduite des trains. In Actes du Colloque international de psychodynamique et de psychopathologie du travail. Paris AOCIP. Pp.149-162

Cruz (de la), MJ., Roche, P. (1990). Trains de vie. Marseille : Matrice.

Dejours, C. (Ed). (1988). Plaisir et souffrance dans le travail. Paris : AOCIP. T1\&2.

Dejours, C. (1993). Travail, usure mentale, suivie d'un addendum théorique De la psychopathologie à la psychodynamique du travail. Paris : Bayard.

Descartes. 1996. Les passions de l'âme. Paris : Flammarion.

Devereux, G. (1970). Essai d'ethnopsychiatrie générale. Paris : Gallimard.

Devereux, G. (1980, première édition 1967). De l'angoisse à la méthode dans les sciences du comportement. Paris : Flammarion.

Dodier, N. (1995). Les hommes et les machines. Paris : Métailié.

Foot, R., Rajoharison, N. (1985). Interfaces homme-machine : les réseaux routier et ferré. Rapport de recherche. Paris : AEROT.

Foot, R. (1990). Le voyageur, l'électricité et le conducteur. In Savoir faire et pouvoir transmettre. Cahier 6. Paris : MSH (Coll. Ethnologie de la France). pp. 133-149.

Foot, R. (1994). RATP, Un corporatisme à l'épreuve des voyageurs. Travail, 31, 63-100.

Foot, R. (1996a). La représentation du voyageur et la RATP, une analyse par les dispositifs de transport. In cahiers de recherche du GIP MI, 70, 15-26.

Foot, R. (1996b). Dans métro, il y a... Travail, 36/37, 27-41. 
Girin, J. Les agencements organisationnels. In F. Charue-Duboc, (Ed). Des savoirs en action. Paris : L'Harmattan. pp. 233-279.

Grumbach T. 1978. La "défense" prud'homale. Versailles : APIL. T1.

Lae, JF. (1991). Le risque, la protection et la sanction. In I. Joseph, JF. Laé, D. Bonniel, Y. Bucas-Français. Généalogie et itinéraires de l'inaptitude. Paris : Réseau 2000/RATP. pp.25-57.

Latour, B. (1993). La clef de Berlin. Paris : La Découverte.

Latour, B. (1994). Une sociologie sans objet? Remarques sur l'interobjectivité. Sociologie du travail, 4, 587-607.

Locqueneaux, L. (1996). Aperçu de la vie quotidienne des conducteurs du métro parisien au travers d'une étude ethnométhodologique et lexicale d'un groupe de conducteurs de la ligne $B$ du RER : le terminus de Denfert Rochereau. Mémoire de DESS d'ethnométhodologie. Université Paris VII-Denis Diderot.

Nathan, T. (1991). De sable, de plomb et de cola. Ethnopsychanalyse des objets actifs. Nouvelle Revue d'Ethnopsychiatrie. 16, 29-51.

Nathan, T. (1993). Fier de n'avoir ni pays, ni amis, quelle sottise c'était. La pensée sauvage.

Nathan, T. (1994). L'influence qui guérit. Paris : Odile Jacob.

Passion, L. (1988). L'accident des Couronnes : technologie et sécurité des transports parisiens au début du $\mathrm{XX}^{\mathrm{e}}$ siècle. In Métropolitain. Paris : Hôtel de Lamoignon. pp. 123-134.

Peslouan (de), F. (1987). Normes de travail, pratiques de production et de consommation. Rapport de recherche. Réseau 2000/Les acteurs du réseau/RATP.

Sigaut, F. (1990). Folie, réel et technologie. Technique \& culture. 15, 167-179.

Simmel, G. (1988). Pont et porte. In La tragédie de la culture et autres essais. Paris :Rivages poche. pp.161-168.

Simmel, G. (1992). Le conflit. Saulxures. Circé.

Supiot, A. (1994). Critique du droit du travail. Paris : PUF.

Thévenot, L. (1985). Les investissements de forme. In Conventions économiques. Cahier du CEE, 29, pp.21-71.

Villoutreix, F. (1990). Modalités d'étude, de conception et d'introduction de systèmes intégrés d'automatismes. Thèse de gestion. Paris : ENSMP.

Mots clés: métro, Météor, conducteur, conduite automatique, suicide, peur, conflits sociaux

\section{Do We Have to Protect Subway from Passengers ? Or Passengers's Apprehension from Engineers and Operators.}

Summary : Actual automatization of Parisan subway raises up a paradox : when operators are suppressed, engineers conceive a subway protected from passengers but leave subways with operators without any protection. This dissymmetrical treatment is stranger because of tacit acquiescence apparently found in the side of operators. Understanding this strange situation implies to 
analyse during a long time the way in which engineers and operators compose their community of actions, through process of automatization of work and conflicts. In the heart of this dynamics, fear of passenger's accident and suicide appears as a decisive element, however it remains on most cases "unexpressed", except under the frame of technical object, during development of a defensive strategy of driving as a profession.

Keywords : Subway, Meteor, automatic driving, suicide, fear, social conflicts.

Hay que proteger el metro de los pasajeros? O el recelo frente al pasajero por parte de ingenieros y conductores.

Resumen : La automatización de actual metro parisino, pone en evidencia un curiosa paradoja moral : desde que se decide suprimir a los conductores, los ingeniores conciben un metro « protegido » de los pasajeros, pero dejan a los conductores sin protectión, en los metros que aun son conducidos por operarios. Esta disimetría en el tratamiento, es aun más bizarra en tanto que pretende encontrar entre los conductores une tácita aquiescencia. La comprensión de esta anormal situación, presupone analizar dentro de un relativamente largo período, de qué manera condutores e ingenieros componene su comundad de acción a través de procesos de automatización, de trabajo y de conflicto. En el corazón de esta dinámica, el miedo al accidente o al suicidio del pasajero que se lanza a las vías, aparece como un elemento determinante. Aun sí la mayor parte del tiempo resulta " inexpresado ", salvo bajo la forma de un objeto técnico, dentro de la elaboración de una estrategia defensiva en el oficio de conducir trenes.

Palabras clave : Metro, Meteoro, conductor, manejo automático, suicidio, mied, conflictos sociales. 